\title{
Offline Data Analysis of Electromagnetic calorimeter trigger in the Belle II Experiment
}

\author{
Haneol Cho, ${ }^{a, *}$ Cheolhun Kim, ${ }^{a 1}$ Y.Unno $^{a 2}$ and ByungGu Cheon ${ }^{a 3}$ \\ ${ }^{a}$ Dept. of Physics, Hanyang University, Seoul 04763, Republic of Korea \\ E-mail: whgksdjf124@hanyang.ac.kr, chkim.hanyang@gmail.com, \\ yunno@post.kek.jp, bgcheon@hanyang.ac.kr
}

We describe the offline data analysis module of Electromagnetic Calorimeter (ECL) trigger in the Belle II experiment. The Belle II experiment at SuperKEKB electron-positron collider in Japan aims to produce $50 \mathrm{ab}^{-1}$ of integrated luminosity. The primary physics motivation is to search for the new physics from heavy quark/lepton flavor decays and dark matter search. To check the ECL trigger performance, we built an offline data monitoring module, Quality Assurance Monitoring (QAM) module. The ECL trigger QAM module is based on the Belle II Aanalysis Framework (basf2). It mainly analyzes the total energy and the cluster energy of Bhabha data skimmed by the Belle II data production group.

40th International Conference on High Energy physics - ICHEP2020

July 28 - August 6, 2020

Prague, Czech Republic (virtual meeting)

\footnotetext{
${ }^{*}$ Speaker
} 


\section{Introduction}

The Belle II [1] experiment aims for an instantaneous luminosity of $8 \times 10^{35} \mathrm{~cm}^{-2} \mathrm{~s}^{-1}$ and an integrated luminosity of $50 \mathrm{ab}^{-1}$ using the SuperKEKB collider [2] at KEK, Japan. We have accumulated data of electron-positron collisions and reached the integrated luminosity of $10 \mathrm{fb}^{-1}$ in December 2019.

The Belle II trigger system [3] comprises the Level 1 hardware trigger (TRG) and the high-level software trigger (HLT). A TRG requirement is a data flow with a maximum trigger rate of $30 \mathrm{kHz}$. The HLT [4] is designed to reduce the event rate of data flow by $10 \mathrm{kHz}$ to a few $\mathrm{kHz}$ as a software trigger.

The ECL detector [1], which contains $8736 \mathrm{CsI}(\mathrm{Tl})$ scintillation crystals in total, comprises barrel, forward, and backward end-cap sections. A trigger cell (TC) is a fast analog trigger signal. The signal is composed of analog signals from neighboring $4 \times 4$ crystals. The ECL trigger provides the total energy, isolated clusters, and Bhabha counting information of electromagnetic particles [5].

\section{ECL trigger QAM module}

We develop the ECL trigger QAM module in order to check the performance of the ECL trigger system. The ECL trigger QAM module includes the inspection system as a function of the experimental run number.

1. Total energy

- We define the total energy as the TC energy addition of an event. The ECL trigger QAM module makes plots of fitted results of the total energy graph (Fig. 1a).

2. Cluster energy sum

- We define the cluster energy sum as the addition of the energy of the two most energetic clusters. Since an electron and a positron take most of the total energy in Bhabha scattering, we expect that the fitted results of the cluster energy sum is similar to total energy's. The ECL trigger QAM module makes plots of fitted results of the cluster energy sum graph (Fig. 1b).

3. TC timing

- We define the TC timing resolution as the time difference of a TC signal from the most energetic TC signal for each event. The ECL trigger QAM module makes plots of fitted results of the TC timing (Fig. 1c).

4. TC anomaly detection

- TC hit is a TC signal larger than $100 \mathrm{MeV}$. The number of TC hits is relatively low if a TC has a problem. We define the TC anomaly as the state in which a TC has fewer TC hits than 10 percent of the average of TC hits. (Fig. 1d). 


\section{Conclusion}

The ECL trigger QAM module could cross-check the ECL trigger performance during the exp10 phase3 (between Nov 8th 19 and Dec 10th 19). We can examine the energy detection performance. We can say that the TC timing resolution is stable. Also, we have confirmed that there is no signal of TC anomaly detection.

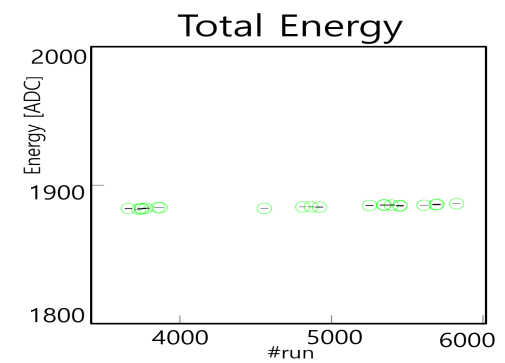

(a) The total energy mean as a function of the experimental run number

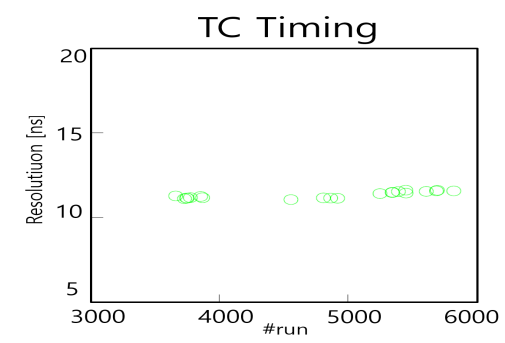

(c) The TC timing resolution as a function of the experimental run number

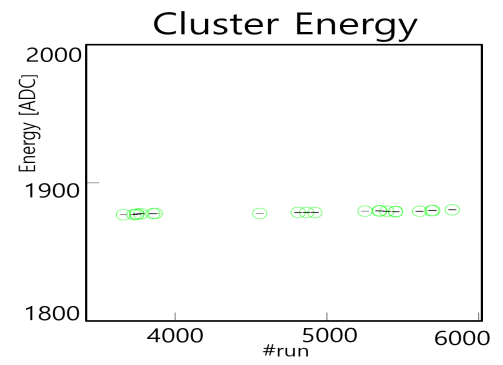

(b) The cluster energy summation mean as a function of the experimental run number

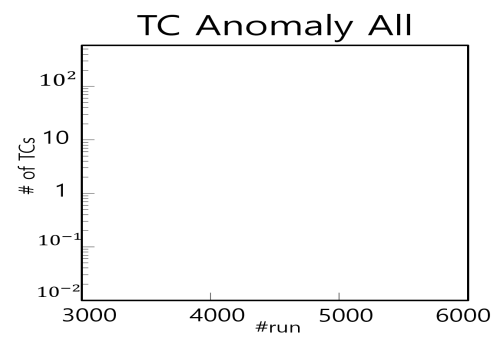

(d) The number of the TC anomaly log scale as a function of the experimental run number

Figure 1: These plots are based on skimmed Bhabha data of the exp10 phase3. We selected runs that include larger than $10 \mathrm{M}$ events. The level of total energy and cluster energy shown on the top panels is similar, reaching the value of about $1900 \mathrm{ADC}$ in Bhabha scattering.

\section{References}

[1] Abe, T., et al. Belle II Technical Design Report. 2010, arXiv:1011.0352.

[2] Ohnishi,, et al. Accelerator design at SuperKEKB. Progress of Theoretical and Experimental Physics 2013, 2013, 03A011.

[3] Iwasaki, Y., et al. Level 1 Trigger System for the Belle II Experiment. IEEE Transactions on Nuclear Science 2011, 58, 1807-1815.

[4] Lee, S., et al. Development of High Level Trigger Software for Belle II at SuperKEKB. Journal of Physics: Conference Series 2011, 331, 022015.

[5] Kim, S., et al. Status of the Electromagnetic Calorimeter Trigger system at the Belle II experiment. Journal of Instrumentation 2017, 12, C09004-C09004. 